\title{
Time to Resolution of Inadvertent Subdural Contrast Injection during a Myelogram: When Can the Study Be Reattempted?
}

\author{
(DD.P. Shlapak, (DD.K. Kim, (DF.E. Diehn, (D).C Benson, (D).T. Lehman, (D).B. Liebo, (D).M. Morris,
} (D) P.P. Morris, (D).T. Verdoorn, and (D)C.M. Carr

\begin{abstract}
BACKGROUND AND PURPOSE: Inadvertent subdural contrast injections can occur during any myelogram. Currently, there are no guidelines defining when residual subdural iodinated contrast will be cleared and no longer interfere with subsequent procedure and imaging. We investigated the time to resolution of subdural contrast using a 2-day lateral decubitus digital subtraction myelogram and associated CT myelogram data in patients undergoing evaluation for spontaneous intracranial hypotension.
\end{abstract}

MATERIALS AND METHODS: Retrospective review of 63 patients with lateral decubitus digital subtraction myelograms from September 4, 2018, to October 1, 2019, was performed. Patients with 2-day lateral decubitus digital subtraction myelograms on 2 consecutive days, with or without a same-day CT myelogram on day 1 and with a same-day CT myelogram on day 2, were included. Patients with next-day CT covering at least the abdomen and pelvis after either-day injection were also included. In cases of subdural injection, next-day CT scans were evaluated for residual subdural contrast.

RESULTS: Of 49 included patients, 5 had subdural injection on day 1, with the second-day CT myelogram available for review. One of these 5 patients had subdural injections on 2 different days and subsequently had chest/abdomen/pelvis CTA a day after the second subdural injection. In all 6 cases of subdural injections, there was complete resolution of subdural contrast on the next-day $\mathrm{CT}$, with the shortest time to resolution of approximately 20.5 hours (range, 20.5-28.5 hours).

CONCLUSIONS: Our study suggests that resolution of inadvertently injected subdural contrast occurs within 1 day, and the myelogram can be reattempted as early as the next day.

ABBREVIATIONS: $C T M=C T$ myelogram; eGFR = estimated glomerular filtration rate; LDDSM = lateral decubitus digital subtraction myelogram

$\mathrm{S}$ ince the introduction of myelography in the mid-1900s, inadvertent subdural contrast injections during myelographic procedures have been called "the anathema to the myelographer" because they can make interpretation of the findings more difficult and potentially inaccurate. ${ }^{1,2}$ Moreover, when a procedure is reattempted, residual subdural contrast could confound the interpretation of the repeat examination. This issue has led some practices to routinely wait several days or even a week before reattempting myelography, which can potentially cause delay in diagnosis and management.

Given the intricacy of the anatomy of the spinal meningeal spaces, by "subdural," we are referring to a cellular interface

Received April 10, 2020; accepted after revision June 15.

From the Department of Radiology, Mayo Clinic, Rochester, Minnesota.

Please address correspondence to Darya Shlapak, MD, MBA, Department of Radiology, Mayo Clinic, 200 1st St SW, Rochester, MN 55905; e-mail:

shlapak.darya@mayo.edu

Indicates article with supplemental on-line table.

http://dx.doi.org/10.3174/ajnr.A6725 between a laminar arachnoid and the inner surface of the duraa so-called "dura-arachnoid interface" or "subdural compartment," composed of neurothelial cells with an amorphous substance. ${ }^{3,4}$ This interface has also been previously described as the medial border of the spinal dura mater, ${ }^{5}$ dural border cell layer, ${ }^{6}$ and subdural mesothelium.?

Suboptimal contrast injections are usually mixed, intrathecal, and subdural, with a variable degree of subdural contrast amount. The timeline to resolution of inadvertent subdural injections has not been evaluated, and currently, there are no guidelines on when a myelogram should or can be reattempted. At our institution, we have been regularly performing lateral decubitus digital subtraction myelograms (LDDSMs) to evaluate CSF leaks since 2018, with alternating sides down on 2 consecutive days, usually immediately followed by a lateral decubitus CT myelogram (CTM). These types of myelograms have a higher rate of subdural injections compared with conventional myelography due to multiple factors, including low CSF pressure, patient positioning, tenting of the dura, and the rate of injection. ${ }^{8}$ Given its consecutive-day nature, this new aspect 

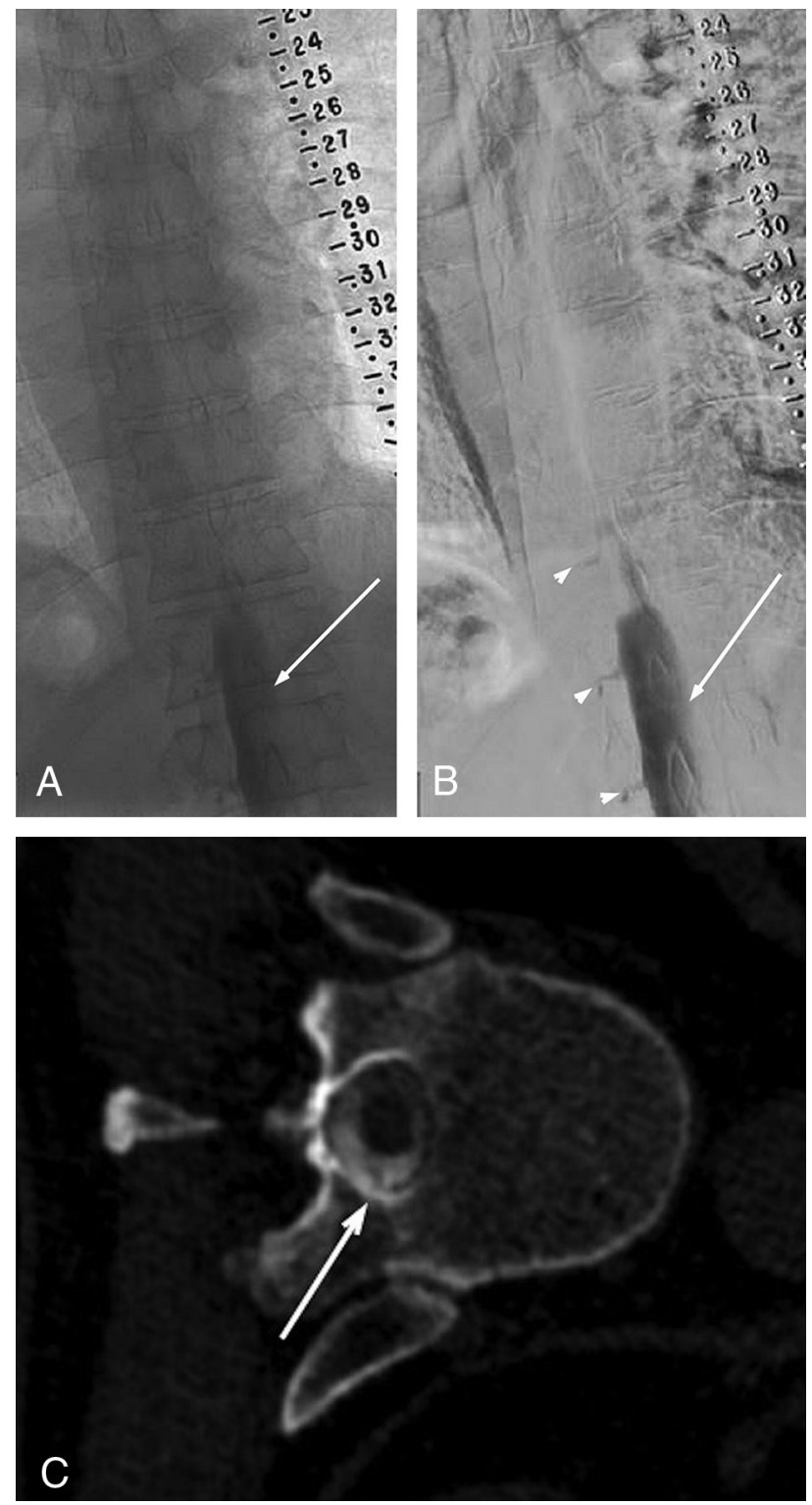

FIG 1. Unsubtracted $(A)$ and subtracted $(B)$ left-side-down LDDSM images on day 1 demonstrate subdural contrast injection at the lower thoracic/upper lumbar levels. Notice nondependent masslike subdural contrast overlying the middle of the osseous spinal canal (solid arrows in $A$ and $B$ ). Given the predominantly subdural injection, only minimal intrathecal contrast is seen filling nerve root sheaths (arrowheads in B). The study was terminated without obtaining a CTM. The next-day CTM following the repeat left LDDSM injection shows no residual subdural contrast at the level of $\mathrm{Tll}$ and a homogeneous appearance of the intrathecal contrast clearly outlining a nerve root traversing the intrathecal space (arrow in C). The time interval between images $A / B$ and $C$ is approximately 20.5 hours.

of modern myelography provides a unique opportunity to evaluate CTM images for resolution of inadvertently injected subdural contrast.

\section{MATERIALS AND METHODS}

This retrospective Health Insurance Portability and Accountability Act-compliant study involving human participants was performed
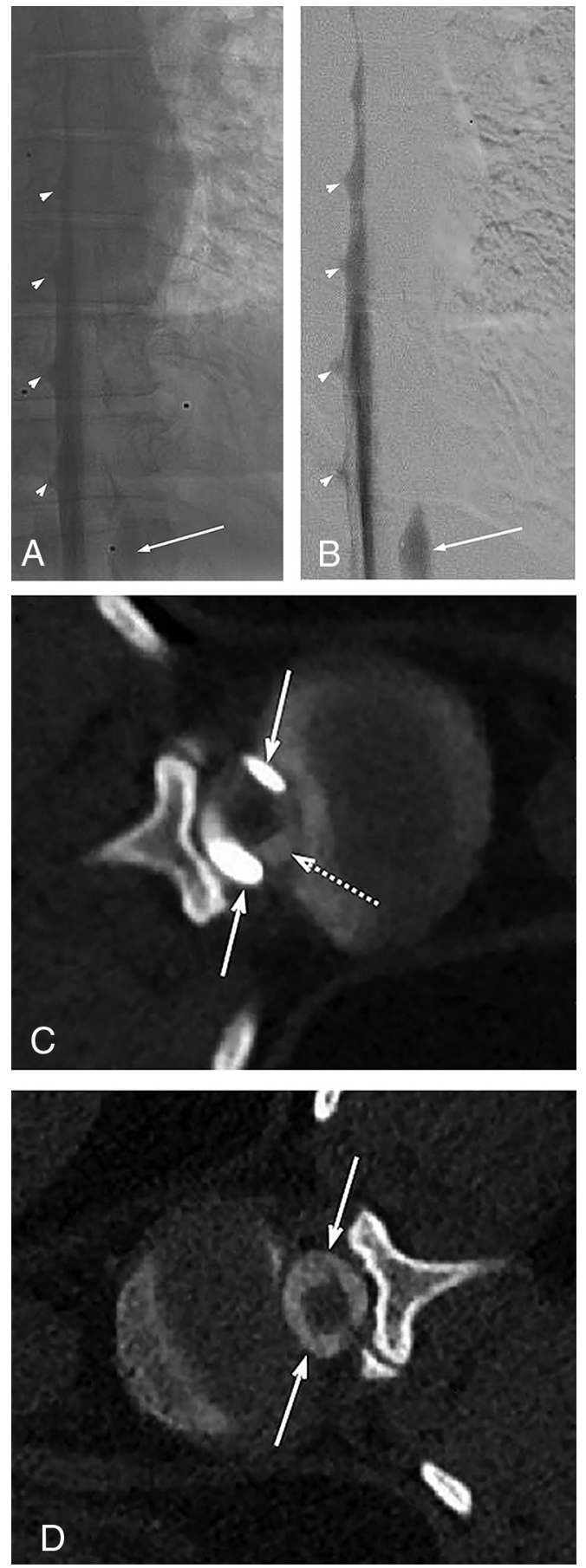

FIG 2. Unsubtracted $(A)$ and subtracted $(B)$ left LDDSM images on day 1 demonstrate subdural contrast injection at the thoracolumbar junction that appears as a nondependent thick contrast column overlying the middle of the osseous spinal canal (solid arrows in $A$ and $B$ ). Given the predominantly intrathecal injection, compare the subdural contrast appearance with the dependently layering menisci of intrathecal contrast filling the nerve root sheaths (arrowheads in $A$ and $B$ ). On the same-day left lateral decubitus CTM (C), subdural contrast appears as nondependent collections (solid arrows in C), more hyperattenuating than the intrathecal contrast (dashed arrow in C). The next-day CTM (immediately following the contralateral right LDDSM) shows no residual subdural contrast at the T12/L1 level (solid arrows in $D$ ). The time interval between images $A / B / C$ and image $D$ is approximately 22.5 hours. 


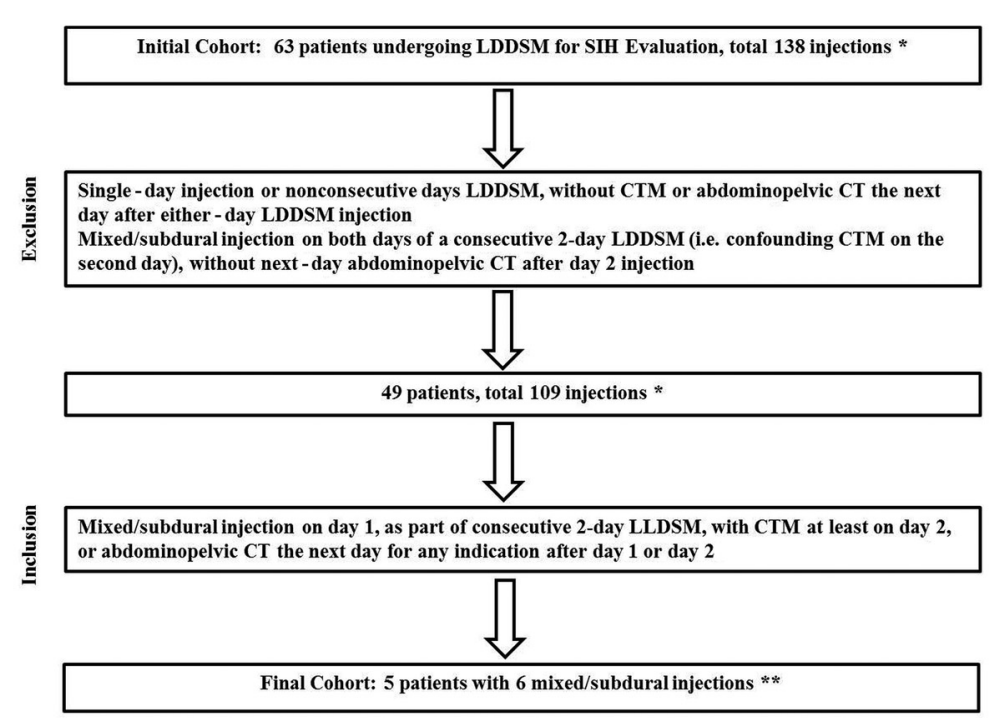

\begin{abstract}
*Number of injections varied per patient, from 1 to 6 , depending on clinical circumstances and need for repeated examinations. **One patient had $2 \mathrm{mixed} / \mathrm{subdural}$ injections: on day 1, with repeat successful intrathecal ipsilateral LDDSM on day 2 (followed by same day 2 CTM), and on day 3 in the contralateral decubitus position. This patient had a CTA of the chest, abdomen, and pelvis the following day (day 4) for artery of Adamkiewicz localization.

SIH, Spontaneous Intracranial Hypotension
\end{abstract}

FIG 3. Patient selection flow chart.

in accordance with the ethical standards of the Institutional and National Research Committee and with the 1964 Declaration of Helsinki and its later amendments or comparable ethical standards. The institutional review board reviewed and approved this study (No. 19-002405). Informed consent was waived.

Patients with symptoms of postural headaches and suspected spontaneous intracranial hypotension were first evaluated for a potential CSF leak by subspecialty neurologists and referred to our service for an LDDSM. The LDDSM technique including patient positioning and needle placement has been recently described. ${ }^{8}$

Subsequent CTMs were performed with the patient in the same lateral decubitus position as the LDDSM covering the skull base to the sacrum. Typically, CTM images were obtained in a single pass with a dual-energy setting $(100 \mathrm{kV}$ and $140 \mathrm{kV})$ and $50-\mathrm{keV}$ monoenergetic reconstructions to increase the sensitivity for subtle contrast leakage. ${ }^{9,10}$ We reconstructed 0.75 - and 3-mm slices from 0.6$\mathrm{mm}$ slices. Next-day CTA of the chest, abdomen, and pelvis in one of the further described subdural contrast injection cases was performed according to routine CTA protocol in arterial and delayed phases from the lung apices to the ischial tuberosities with 1- and 2-mm slices reconstructed from $0.6-\mathrm{mm}$ slices.

We reviewed imaging studies and reports of 63 patients who underwent LDDSMs at our institution as part of an evaluation for spontaneous intracranial hypotension, without or with same-day concurrent CTM from September 4, 2018, to October 1, 2019, for 138 separate contrast injections. Demographic data including patient sex and age at the time of LDDSM were recorded. Additionally, the estimated glomerular filtration rate (eGFR) in patients with mixed, subdural, and intrathecal contrast injections around the time of LDDSM if available was also recorded.

To be included in the final study group, patients had to complete both days of the LDDSM (left and right side down) performed on 2 consecutive days, with a CTM immediately following the LDDSM at least on the second day of the 2-day study. Additionally, patients who had the next-day CT covering at least the abdomen and pelvis after either-day injection or after a single-day study were also included.

Unsubtracted and subtracted LDDSM and CTM images were reviewed for the presence of subdural contrast. The relative degree of intrathecal-versus-subdural contrast was subjectively estimated on the basis of visual inspection of the images and graded as entirely intrathecal, predominantly subdural, or predominantly intrathecal, with "predominantly" qualitatively referring to $>50 \%$ of the total volume of injected contrast. Subdural contrast was usually evident on unsubtracted and subtracted LDDSM images as a nondependent masslike thick column of contrast overlying the middle of the osseous spinal canal, rather than dependently layering menisci of intrathecal contrast filling the nerve root sheaths (Figs 1 and $2 A$ and $-B$ ). On subsequent lateral decubitus CT myelograms, subdural contrast appeared as a nondependent collection or a more globular-like area of contrast, more hyperattenuating and confined than contrast seen intrathecally (Fig 2C).

\section{RESULTS}

Of 63 patients with 138 injections, 49 patients with 109 injections met the inclusion criteria and composed the final study group: 31 women and 18 men, with an average age of 56 years (range, 2878 years). A flow chart demonstrating the selection of the final patient study cohort is presented in Fig 3. Of these 49 patients, 5 had a mixed (subdural and intrathecal) injection on the first day of the study: 3 women and 2 men, with the average age of 59 years (range, 36-78 years). One of these 5 patients with subdural injection on the first day (injection 2 in the On-line Table) had a repeat successful intrathecal ipsilateral LDDSM on the second day but then again had a mixed injection on the third day in the contralateral decubitus position (injection 3 in the On-line Table). This patient had a CTA of the chest, abdomen, and pelvis on the following day (fourth day) for artery of Adamkiewicz localization. Therefore, in total, there were 5 patients with 6 mixed injections who had next-day CT images available for evaluation. Of the 5 patients with subdural injections, 3 had an eGFR of $>60$ (injections 1-4 in the On-line Table), 1 patient had an eGFR of 51 (injection 5), and 1 had no renal function panel available around the time of the LDDSM (injection 6).

All 5 second-day CTMs and a next-day CTA in injection 3 demonstrated complete resolution of subdural contrast injected on the previous day (Figs 1 and 2). The shortest time between 
the LDDSM subdural injection and the next-day CT was approximately 20.5 hours. Resolution of the other 5 subdural injections was confirmed on the next-day CT images after approximately $22.5,24,26,26$, and 28.5 hours after the previous-day LDDSM injection, respectively, with a mean time interval of 24.6 hours.

Patient position, total contrast amount/type, relative degree of subdural contrast, needle gauge, postinjection imaging, outcomes, and time to subdural contrast resolution per next day CT are summarized in the On-line Table.

Patients with mixed injections 1, 5, and 6 in the On-line Table did not have a spinal CSF leak on either day of the study on LDDSM or CTM images. Patient 2 (injection 2 in the Online Table) did not have a left CSF leak on the first day of mixed injection or next-day repeat left LDDSM or CTM images but had an identifiable right T6/T7 CSF-venous fistula on the right LDDSM despite another mixed injection (injection 3 in the On-line Table). Subdural contrast in injection 3 extended from the lowest visualized level on the LDDSM of L1 to the T7 inferior endplate level and from the S1 to T7 superior endplate on the same-day right-side-down CTM images.

Finally, the patient with a mixed injection 4 in the On-line Table did not have a left spinal CSF leak on the first-day left LDDSM or same-day CTM images at the time of the mixed injection. However, on the next-day right LDDSM and the same-day right-side-down CTM, there was an identifiable right CSF leak presumably due to a dural tear at L2/L3. Subdural contrast from the mixed injection on day 1 on the left LDDSM extended from the lowest visualized level of L2 to T7 and on the same-day leftside-down CTM from L3 to T4.

\section{DISCUSSION}

Our study suggests that subdural contrast resolves within approximately 24 hours. While mixed intrathecal and subdural injections can still be diagnostic, in nondiagnostic cases due to predominantly subdural injection, our findings suggest that the examination can be repeated as early as the next day. Because there are currently limited data and lack of consensus as to when a myelogram can be reattempted after subdural injection, leading to many practices waiting days to weeks before repeat examination, our study results can facilitate an earlier reattempt at performing the myelogram and avoiding further delay in diagnosis.

Although these findings are applicable to any type of myelography using iodinated contrast, they are particularly helpful for proceduralists performing LDDSMs. As noted by Kim et $\mathrm{al}^{8}{ }^{8}$ subdural injections are more common during these examinations due to multiple factors, including low CSF pressure, patient positioning, tenting of the dura, and the rate of injection.

The mechanism of clearance of subdural contrast remains unclear and is not addressed by the present study. A case report by Yi et $\mathrm{al}^{11}$ described spontaneous resolution of an idiopathic lumbar subdural hygroma after approximately 2 weeks, documented by a CT myelogram and lumbar spine MR imaging. On the basis of 65 radiographic studies in the mid-1900s of iophendylate distribution being injected in the subdural space, Hugh, ${ }^{12}$ in 2010, suggested that contrast could be rapidly reabsorbed by a rich lymphatics network.

Of 5 patients with subdural injections in our study, 3 had an eGFR of $>60$, one had an eGFR of 51, and 1 had no renal function panel available around the time of the LDDSM. Although we suspect that renal function is unrelated to subdural contrast clearance, more research with larger study groups and more variable renal function is needed.

Additionally, given resolution of subdural contrast in all 6 cases of mixed injections on the next-day CT images with or without a CSF leak, it seems unlikely that close approximation of the CSF leak site to subdural contrast would impact its time to resolution.

Our study has several additional limitations, including its small number of patients with mixed contrast injections who had next-day CT images available for review. Additionally, the results of a mixed injection, even predominately subdural, might not apply to a full subdural injection.

Finally, all patients in our study group had a presumptive diagnosis of spontaneous intracranial hypotension, and the results may not be generalizable to other patient populations. For example, patients with severe spinal canal stenosis or prior spine surgery may have alteration of subdural contrast clearance.

Future work could include larger retrospective studies by centers performing a high volume of 2-day LDDSMs with concurrent CTMs confirming next-day resolution of subdural contrast as well as evaluating the diagnostic yield of mixed injections. Prospective studies could include obtaining a limited CT within 24 hours in cases of mixed injection for various indications and different types of myelograms.

\section{CONCLUSIONS}

Our study suggests that subdural contrast inadvertently injected during a myelographic procedure resolves after approximately 24 hours and as early as after 20.5 hours. A myelogram could be reattempted as early as the next day, without concerns that residual subdural contrast could interfere with diagnostic quality of the repeat examination.

\section{REFERENCES}

1. Camp JD. Contrast myelography past and present. Radiology 1950;54:477-506 CrossRef

2. Schultz EH Jr, Brogdon BG. The problem of subdural placement in myelography. Radiology 1962;79:91-96 CrossRef Medline

3. Collier $\mathrm{CB}$. The intradural space: the fourth place to go astray during epidural block. Int J Obstet Anesth 2010;19:133-41 CrossRef Medline

4. Reina MA, Collier CB, Prats-Galino A, et al. Unintentional subdural placement of epidural catheters during attempted epidural anesthesia: an anatomic study of spinal subdural compartment. Reg Anesth Pain Med 2011;36:537-41 CrossRef Medline

5. Waggener JD, Beggs J. The membranous coverings of neural tissues: an electron microscopy study. J Neuropathol Exp Neurol 1967;26:41226 CrossRef Medline

AJNR Am J Neuroradiol 41:1958-62 Oct 2020 www.ajnr.org 
6. Alcolado R, Weller RO, Parrish EP, et al. The cranial arachnoid and pia mater in man: anatomical and ultrastructural observations. Neuropathol Appl Neurobiol 1988;14:1-17 CrossRef Medline

7. Orlin JR, Osen KK, Hovig T. Subdural compartment in pig: a morphologic study with blood and horseradish peroxidase infused subdurally. Anat Rec 1991;230:22-37 CrossRef Medline

8. Kim DK, Brinjikji W, Morris PP, et al. Lateral decubitus digital subtraction myelography: tips, tricks, and pitfalls. AJNR Am J Neuroradiol 2020;41:21-28 CrossRef Medline

9. Postma AA, Das M, Stadler AA, et al. Dual-energy CT: what the neuroradiologist should know. Curr Radiology Rep 2015;3:16 CrossRef Medline
10. Shuman WP, Chan KT, Busey JM, et al. Dual-energy CT a ortography with $50 \%$ reduced iodine dose versus single-energy CT aortography with standard iodine dose. Acad Radiol 2016;23:611-18 CrossRef Medline

11. Yi CK, Biega TJ, Burgos RM. Spontaneous resolution of idiopathic lumbar subdural hygroma on CT myelography and lumbar spine MRI. BMJ Case Rep 2014;2014:bcr2014206223 CrossRef Medline

12. Hugh AE. The subdural space of the spine: a lymphatic sink? Myodil's last message. Clin Anat 2010;23:829-39 CrossRef Medline 


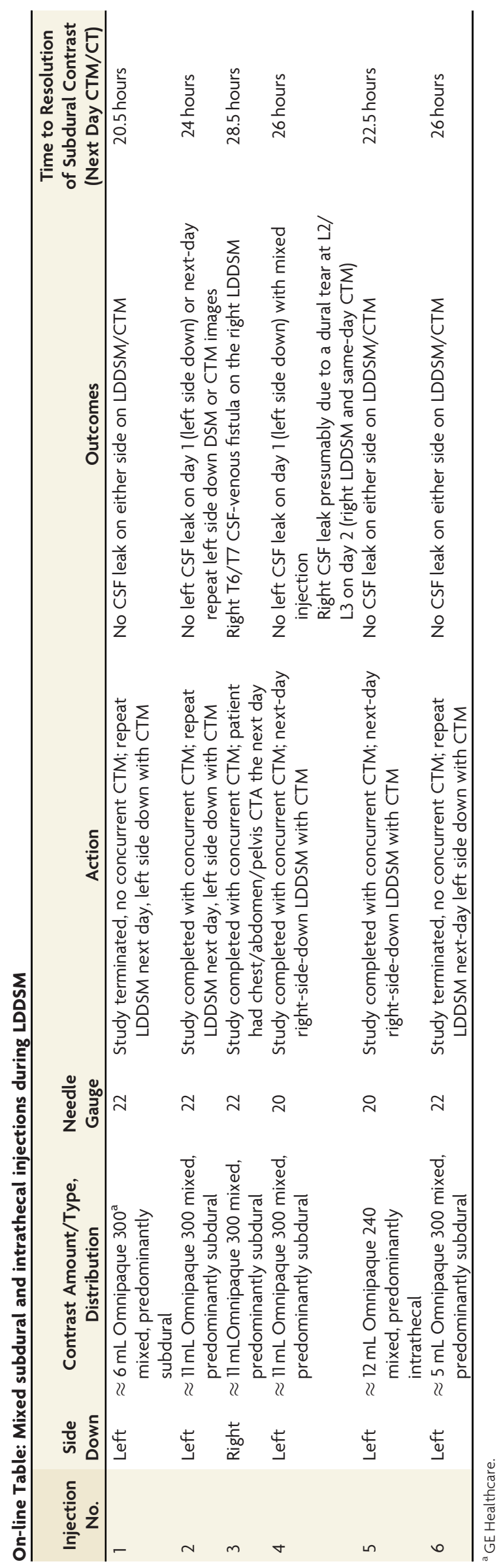

